DTP-97/02

March, 1997

\title{
Unquenching the scalar glueball
}

\author{
M. Boglione ${ }^{1,2}$ \\ and \\ M.R. Pennington ${ }^{1}$ \\ ${ }^{1}$ Centre for Particle Theory, University of Durham \\ Durham DH1 3LE, U.K. \\ ${ }^{2}$ Dipartimento di Fisica Teorica, Università di Torino and \\ INFN, Sezione di Torino, Via P. Giuria 1, 10125 Torino, Italy
}

\begin{abstract}
Computations in the quenched approximation on the lattice predict the lightest glueball to be a scalar in the $1.5-1.8 \mathrm{GeV}$ region. Here we calculate the dynamical effect the coupling to two pseudoscalars has on the mass, width and decay pattern of such a scalar glueball. These hadronic interactions allow mixing with the $q \bar{q}$ scalar nonet, which is largely fixed by the well-established $K_{0}^{*}(1430)$. This non-perturbative mixing means that, if the pure gluestate has a width to two pseudoscalar channels of $\sim 100 \mathrm{MeV}$ as predicted on the lattice, the resulting hadron has a width to these channels of only $\sim 30 \mathrm{MeV}$ with a large $\eta \eta$ component. Experimental results need to be reanalyzed in the light of these predictions to decide if either the $f_{0}(1500)$ or an $f_{0}(1710)$ coincides with this dressed glueball.
\end{abstract}


QCD without quarks, the gauge theory of gluon interactions, predicts a spectrum of hadrons quite unlike the world experiments so clearly reveals. The imprint of this pure gauge world would be a spectrum of glueballs, the lightest of which would be stable with scalar quantum numbers. Lattice calculations have now reached sufficient precision to predict this scalar glueball has a mass of $1740 \pm 71 \mathrm{MeV}$ [1] or $1550 \pm 50$ $\mathrm{MeV}$ [3] depending on how the lattice data are analysed. More recently, using improved lattice actions, Morningstar and Peardon [ [ [] give results that allow a central mass of $1600 \mathrm{MeV}$ to be deduced. Moreover, the IBM group [2] have calculated its coupling to two pseudoscalars would give a width of $100 \mathrm{MeV}$ for the scalar, if it could decay to these. How this state of the quarkless world would appear in the real world is what this letter is about.

For more than thirty years we have understood that (most) hadrons are closely connected to the states of underlying quark multiplets: the vector and tensor mesons and the baryon octet and decuplet being the best known examples. The properties of these states are determined by the bound state dynamics of quarks. This fixes their masses and decays. Thus, it is natural that the $f_{2}(1270)$ and $f_{2}(1525)$ decay predominantly to $\pi \pi$ and $K \bar{K}$ respectively. Indeed, it is the composition of the underlying state that determines its decays, while the masses of the open channels are incidental. However, as emphasised by Tornqvist [7], scalar mesons may be rather different. They couple far more strongly and are far more sensitive to the opening of thresholds, particularly those with S-wave interactions.

In the quark model we build $0^{++}$mesons by having a $q \bar{q}$ system with spin one and a unit of orbital angular momentum. The lightest of these is expected in the $1-2 \mathrm{GeV}$ region, in which the scalar glueball also occurs. The isospin zero $q \bar{q}$ members are expected to mix with the gluestate to give the hadrons we observe. While a simple quark model of mixing using wholly perturbative methods may be appropriate for other quantum numbers, the properties of scalars makes such calculations far too simplistic. Instead the mixing is highly non-perturbative and requires a more detailed discussion of the hadron propagators. The natural vehicle for this is the appropriate Schwinger-Dyson equation. To calculate this, we assume the coupling to two pseudoscalar channels 
controls the dynamics and hence the mixing.

The calculation. We begin with the quenched approximation, which delivers a bare state of mass $m_{0}$ with point couplings $g_{i}$ to each pseudoscalar channel. Reflecting the spatial extent of hadrons, these couplings are multiplied by form factors $F\left(k_{i}^{2}\right)$, where $k_{i}$ is the channel's c.m. 3-momentum and

$$
F\left(k_{i}^{2}\right)=\exp \left[-k_{i}^{2} /\left(2 k_{0}^{2}\right)\right]
$$

$k_{0}$ is related to the interaction radius, which is taken to be between 0.5 and $1 \mathrm{fm}$. As a consequence of chiral dynamics, the couplings to two pseudoscalars have an Adler zero at $s_{A, i}$, so the full coupling $G_{i}$ is given by

$$
G_{i}^{2}=g_{i}^{2}\left(s-s_{A, i}\right) F^{2}\left(k_{i}^{2}\right)
$$

The effect of unquenching is to dress the bare bound state propagators. This gives them imaginary parts that are the prerequisite for decay. The Dyson summation of Fig. 1 gives

$$
m^{2}(s)=m_{0}^{2}+\Pi(s)
$$

where $\Pi(s)$ is assumed to be dominated by the two pseudoscalar meson loops.

Its imaginary part is simply related to the couplings of Eq. (2) by the appropriate phase-space factor

$$
\operatorname{Im}_{i}(s)=-\rho_{i}(s) G_{i}^{2}(s) \theta\left(k_{i}^{2}\right),
$$

where $\rho_{i}(s)=k_{i}(s) / \sqrt{s}$. The real part of $\Pi(s)$ is now fixed by an unsubtracted dispersion relation - unsubtracted because hadrons have a spatial extent making only

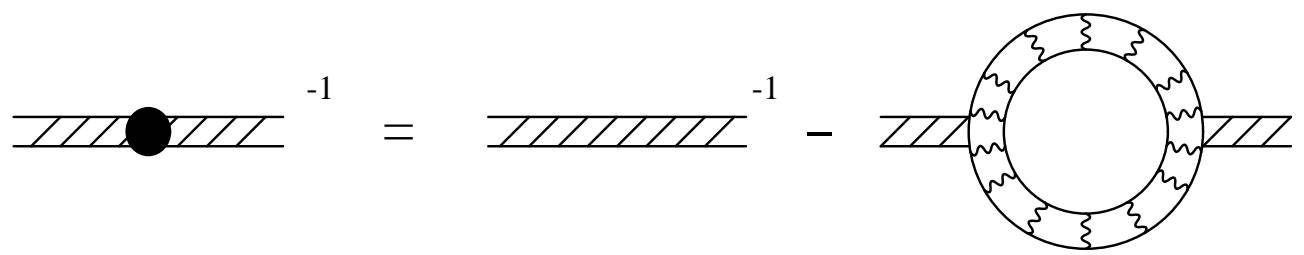

Figure 1: The bare bound state propagator is dressed by hadronic interactions. The dot signifies the dressed propagator. The loop is of $q \bar{q}$ pseudoscalars: the wiggly lines are to enphasize these too are bound states. 
a finite number of hadronic channels relevant. Thus

$$
\operatorname{Re} \Pi(s)=\frac{1}{\pi} \mathcal{P} \int_{s_{t h}}^{\infty} d s^{\prime} \frac{\operatorname{Im} \Pi\left(s^{\prime}\right)}{s^{\prime}-s}
$$

with

$$
\operatorname{Im} \Pi(s)=\sum_{i} \operatorname{Im}_{i}(s)
$$

where we sum over all two pseudoscalar modes, e.g. $K \pi, K \eta, K \eta^{\prime}$ for the $I=1 / 2$ channel. The effect of these meson loops is to produce a propagator

$$
\Delta=\frac{1}{m^{2}(s)-s}=\frac{1}{m_{0}^{2}+\Pi(s)-s},
$$

where the pole is now in the complex energy plane (with reflections on each unphysical sheet generated by the thresholds to which the state couples). It is the position of this pole at $s=m_{\text {pole }}^{2}-i m_{\text {pole }} \Gamma_{\text {pole }}$ that defines the mass, $m_{\text {pole }}$, and width, $\Gamma_{\text {pole }}$, of the hadron.

This is the formalism that applies in the $I=1 / 2$ and 1 scalar channels, where the ground state quark model gives one bare 'seed'. However, in the $I=0$ channel we have more bare states: two ground state $q \bar{q}$ 's and the glueball. The philosophy here is that all mixing occurs through communicating hadronic final states. Thus the bare $q \bar{q}$ nonet is assumed to be ideally mixed. This is in keeping with the notion that the $\phi$, for example, decays to $\rho \pi$ through hadron interactions, eg. $\phi \rightarrow K \bar{K} \rightarrow \rho \pi$, and not through any non-hidden-strangeness at the bare level. With 3 bare states $(n \bar{n}, s \bar{s}$, $g g$ ) of the same quantum numbers, the propagator of Eq. (7) becomes a $3 \times 3$ matrix. The hadrons are then the eigenstates of this matrix. To determine these requires a diagonalization. This diagonalization accounts for the mixing between states of the same quantum numbers.

The input for the bare seeds is as follows.

- There is an ideally mixed $q \bar{q}$ multiplet. The mass of the non-strange members is $m_{0}(n \bar{n})$, and the mass splitting is determined by the extra mass of the strange quark, where $\Delta m_{s} \simeq 100 \mathrm{MeV}$ from standard phenomenology. The couplings of these bare bound states to two pseudoscalars are assumed to be related by 
$S U(3)_{f}$ symmetry, so that in fact there is effectively only one overall coupling for the nonet, $\gamma$. Similarly, the cut-off $k_{0}$ in the form factor of Eq. (1) is assumed universal. The parameters $m_{0}, \gamma$ and $k_{0}$ are to be determined.

- The quenched gluestate has mass $m_{0}(g g)$ specified by lattice calculations. The coupling $\gamma^{\prime}$ is arranged to give a width of the unmixed glue state, to the sum of the two pseudoscalar channels we consider, of $(108 \pm 29) \mathrm{MeV}$ as computed by the $I B M$ group [2]. The coupling to individual channels is assumed to be according to one of two schemes, either

(a) the bare state is an $S U(3)_{f}$ singlet, or

(b) the bare state has couplings as found by the $I B M$ group [2] on the lattice.

The size of the effect of turning on hadron loops can be understood qualitatively. For S-wave decays, each threshold produces a jump, Eq. (4), in the imaginary part of the mass function directly related to the strength of the coupling to the opening threshold with a corresponding cusp in the real part. If the coupling strength gives a width of $100 \mathrm{MeV}$, for example, then this may shift the mass of any bare state that is within $100 \mathrm{MeV}$ or so of this threshold by roughly $100 \mathrm{MeV}$ and generally towards this threshold. This naturally has the effect of making the scalar mesons appear close to strongly coupled thresholds [5], potentially attracting the $a_{0}(980)$ and $f_{0}(980)$ to $K \bar{K}$ threshold, for example, if the parameters are suitable.

The $I=1 / 2$ and 1 sectors. Though all the light scalars feature in our discussion, the major experimental ingredient in determining $m_{0}, \gamma, k_{0}$ is the well established $K_{0}^{*}(1430)$, which from the $P D G$ tables 6 has

$$
m=(1429 \pm 6) \mathrm{MeV} ; \Gamma=(287 \pm 23) \mathrm{MeV} .
$$

That this $I=1 / 2$ state is a member of the lightest $q \bar{q}$ nonet is uncontroversial. In contrast, whether the $a_{0}(980)$, or a possible $a_{0}(1430)$, belongs to this same multiplet is a priori not so clear. 
In a closely related analysis [7], Tornqvist has determined the parameters $m_{0}, \gamma, k_{0}$ with great precision from the $K \pi$ phase shift that reflects the presence of the $K_{0}^{*}(1430)$. To do this he assumes the $K \pi$ scattering amplitude is pole dominated. This not only requires the denominator of any pseudoscalar scattering amplitude to be given by the propagator of Eq. (7), but assumes the numerator has a most specific form. This is a very special unitarization that is far from the most general, in particular it lacks crossing-symmetry. We do not enter here into the controversy [8] about what effect Tornqvist's strong assumptions have on the existence of a light $\sigma$ resonance, suffice it to say that the treatment by Roos and Tornqvist [9] neglects the cross-channel dynamics that would appear in the numerator of an $N / D$ analysis. Here it is only the $D$-function that enters our discussion.

Since we consider just the denominators of the scalar amplitudes, we find a range of values of the parameters $m_{0}, \gamma, k_{0}$ to be consistent with the strange state's mass and width, Eq. (8) - a range that includes Tornqvist's numbers. With central values we show in Fig. 2 the real and imaginary parts of the $I=1 / 2$ mass function $m^{2}(s)$, together with that for $I=1$, the parameters of which are also implicitly constrained by Eq. (8). This fixes the bare $n \bar{n}$ state to be $\sim 1420 \mathrm{MeV}$. It is only the pole in a propagator that fixes the parameters of the state and, of course, it is only this pole position that is process independent. Consequently, we have to continue Eqs. (5, 6, (7) into the complex $s$-plane onto the appropriate unphysical sheets. The scalars being generally broad and the effect of thresholds marked, the mass functions vary strongly as one moves into the complex $s$-plane. The sheets are specified by the signs of the $\operatorname{Im} k_{i}$, as there is a bifurcation at each threshold $i$. In Table I we give the mass and width $m_{\text {pole }}, \Gamma_{\text {pole }}$ on the nearest unphysical sheet.

The $\mathbf{I}=\mathbf{0}$ sector. Turning to the $I=0$ sector, the masses and couplings of the bare $q \bar{q}$ nonet members are now fixed. The bare glueball mass $m_{0}(g g)$ is taken to be either $1600 \mathrm{MeV}$ from Morningstar and Peardon [4] or $1740 \mathrm{MeV}$ from the IBM group [2]. Unquenching by first turning on the pseudoscalar loops of Fig. 1 gives the complex mass function shown in Fig. 3. $I_{0}, I I_{0}$ correspond to the pure glueball having, respectively, either $S U(3)_{f}$ singlet couplings or the pattern computed by the $I B M$ group that favors 
the higher mass channels. It is by making the imaginary part of this mass function correspond to a width of $\sim 100 \mathrm{MeV}$ at the gluestate mass that fixes the coupling $\gamma^{\prime}$. There are, of course, analogous mass functions for the $n \bar{n}$ and $s \bar{s}$ quark model scalars. To find the physical hadrons, we have to diagonalize the $3 \times 3$ mass matrix formed from the $n \bar{n}, s \bar{s}$ and $g g$ states. In physical terms, this allows the quark and glue configurations to mix through their common communicating channels : $\pi \pi, K \bar{K}, \eta \eta$, $\eta \eta^{\prime}$ and $\eta^{\prime} \eta^{\prime}$. Importantly, as noted earlier by Tornqvist [7], with the parameters of the quark multiplet fixed largely by the $K_{0}^{*}(1430)$, the ground state isoscalars are naturally the $f_{0}(980)$ and $f_{0}(1300)$. A ground state $s \bar{s}$ scalar up at $1700 \mathrm{MeV} 10$ is alien to the non-perturbative mixing computed here. The resulting $I=0$ pole positions are given in Table II, again for central values of the parameters. The presence of the gluestate has little effect on these predominantly $q \bar{q}$ states.

However, as seen in Fig. 3, the mixing has an appreciable effect on the gluestate. Its coupling to the $n \bar{n}$ and $s \bar{s}$ states dramatically reduces the width of the unquenched hadron - by how much depends on its mass and coupling pattern. For an underlying flavor singlet, the width is down from $100 \mathrm{MeV}$ to $\sim 30 \mathrm{MeV}$ if $m_{0}(g g)=1740 \mathrm{MeV}$ and to only a few $\mathrm{MeV}$ if $m_{0}(g g)=1600 \mathrm{MeV}$.

With this suppression of the couplings to the decay channels, the real part of the mass function, labelled $I, I I$ in Fig. 3 becomes almost independent of $s$. The suppression is most appreciable for a lighter glueball with $S U(3)_{f}$ couplings. This is because the

\begin{tabular}{|c|c|c|c|}
\hline Resonance & $m_{\text {pole }}$ & $\Gamma_{\text {pole }}$ & sheet \\
\hline \hline$K_{0}^{*}(1430)$ & 1445 & 334 &,,--+ \\
\hline$a_{0}(980)$ & 1082 & 309 &,,-++ \\
\hline$f_{0}(980)$ & 1006 & 54 &,,-++ \\
\hline$f_{0}(1300)$ & 1203 & 361 &,,--- \\
\hline
\end{tabular}

Table 1: Masses and widths of the scalar nonet members in $\mathrm{MeV}$, with $\gamma=1.15$. The sheets are defined by the signs of $\operatorname{Im} k_{i}$ for $i=1,2,3$, as appropriate. 

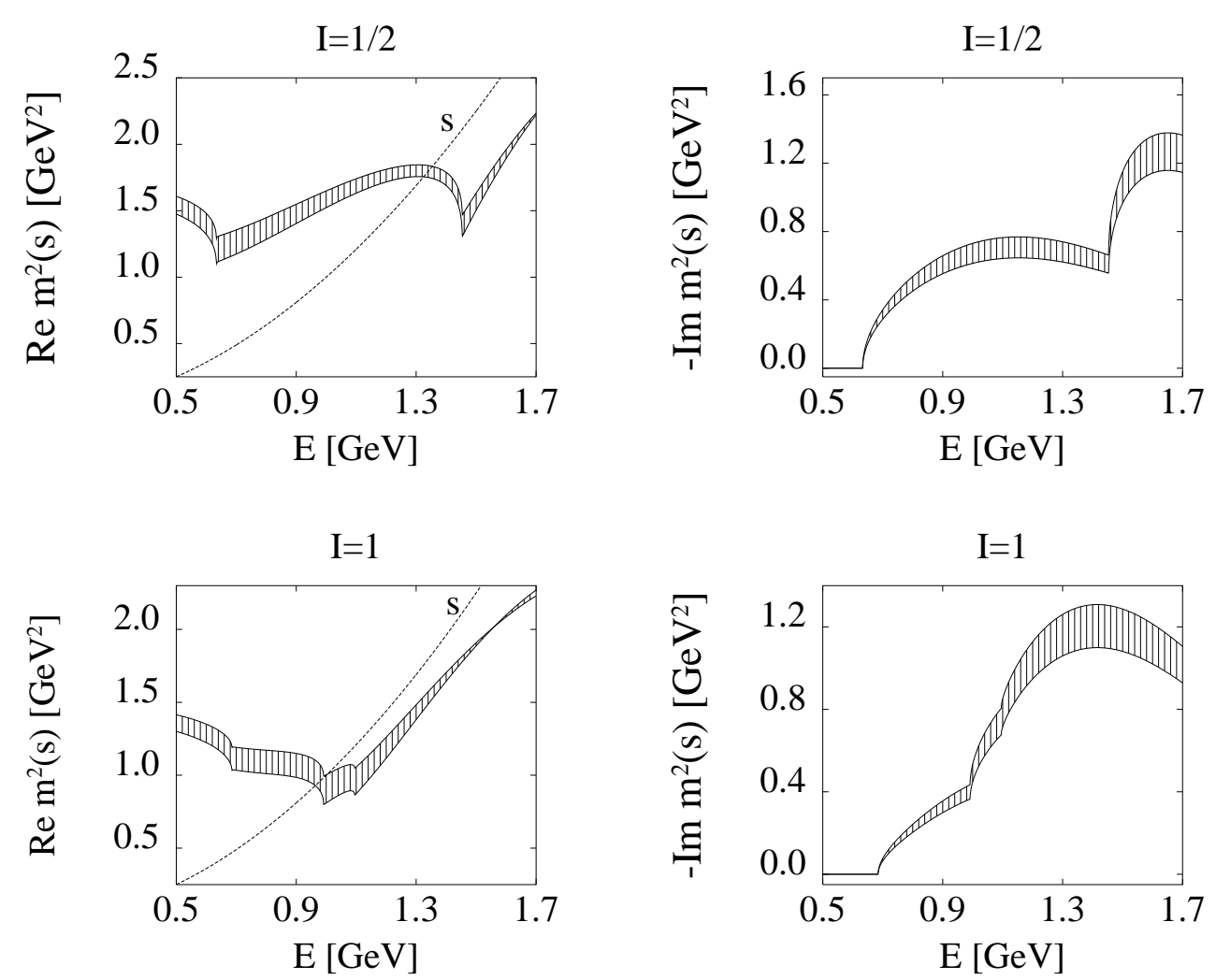

Figure 2: The real and imaginary parts of $m^{2}(s)$ as functions of $E=\sqrt{s}$ for the $I=1 / 2$ and 1 propagators. Here $m_{0}=1420 \mathrm{MeV}$ and $\gamma$ is taken to be between 1.1 and 1.2. The upper end of each band has $\gamma=1.1$ and the lower $\gamma=1.2$.

mixing with quark states occurs most through $\pi \pi$ and $K \bar{K}$ intermediate states. The $I B M$ pattern of couplings favors the heavier pseudoscalars and the width suppression is consequently less. With the bare $n \bar{n}$ state being at $1420 \mathrm{MeV}$ and the $s \bar{s}$ at 1620 $\mathrm{MeV}$, a gluestate at $1600 \mathrm{MeV}$ interacts quite differently from one with a bare mass of $1740 \mathrm{MeV}$. In Table III we give the corresponding widths of the mainly gluish hadron to each two pseudoscalar channel. Notice that there is no large width to $K \bar{K}$. Indeed, it is the $\eta \eta$ decay mode that provides the largest width. These are the predictions that experiment has to check.

Experiment in fact delivers two potential candidates for the unquenched glueball : $f_{0}(1500)$ with a width of $(120 \pm 19) \mathrm{MeV}$ seen by the Crystal Barrel experiment in $p \bar{p}$ annihilation in several different channels [11], and the $f_{J}(1710)$ with even spin and width of $(175 \pm 9) \mathrm{MeV}$, first identified in $J / \psi$ radiative decays [12]. Our results show that there is prima facie difficulty in identifying either of these with the glueball. 


\begin{tabular}{|l|c|c|c|c|}
\hline Couplings & $m_{0}(g g)$ & $m_{\text {pole }}$ & $\Gamma_{\text {pole }}$ & sheet \\
\hline \hline$S U(3)_{f}$ singlet & 1600 & 1591 & 3 &,,--+ \\
\hline$S U(3)_{f}$ singlet & 1740 & 1715 & 32 &,,--+ \\
\hline$I B M$ & 1600 & 1589 & 22 &,,--- \\
\hline$I B M$ & 1740 & 1715 & 28 &,,--- \\
\hline$I B M+4 \pi$ channel & 1600 & 1564 & 108 &,,,-+-- \\
\hline$I B M+4 \pi$ channel & 1740 & 1706 & 127 &,,,---- \\
\hline
\end{tabular}

Table 2: Mass and width of the glue-state in $\mathrm{MeV}$ corresponding to various choices of bare mass and couplings. The sheets are defined as in Table I.

Notice that the coupling pattern computed by the IBM group [2] for a quenched (nondecaying) glueball does not survive strong mixing with the other scalars and their decay channels. The resulting hadron, rather than coinciding with the $f_{0}(1710)$ of Lindenbaum and Longacre [13], has a large $\eta \eta$ decay width much more like the GAMS $G(1590)$ state [14. The latest PDG tables [6] identify this with the WA91 $f_{0}(1450)$ [15] and the Crystal Barrel $f_{0}(1500)$ [16], despite largely contradictory decay information - agreed with relatively small statistics from GAMS.

There is little doubt that the opening of multi-pion channels in the $1400 \mathrm{MeV}$ region, which we have so far neglected, can have a marked effect on the gluestate, while changing the ground state quark states rather little. To mimic these decays, we can arrange for a $4 \pi$ channel [16] to enhance the dressed gluestate to a $\sim 120 \mathrm{MeV}$ total width. This leaves its partial widths to two pseudoscalars as in Table III. Then the larger couplings shift the mass of the "unquenched" glueball downwards and this becomes much more like the $f_{0}(1500)$ with poles as in Table II.

Conclusions. The present work yields definite predictions for the decay pattern of the dressed glueball to be compared with experiment. The analysis of experiment is however not without its ambiguities. Consequently, the challenge is to perform a 

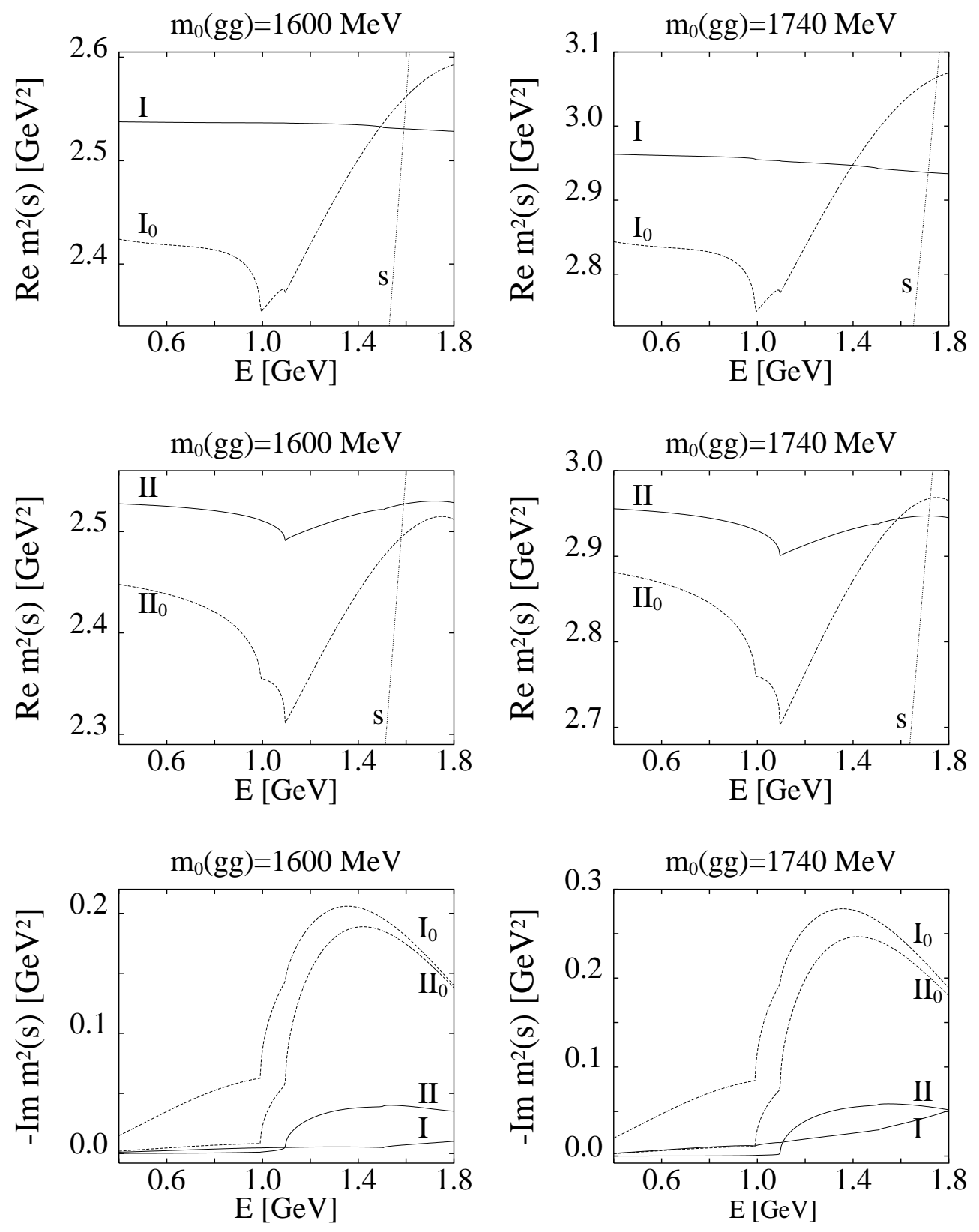

Figure 3: The real and imaginary parts of the glue-state $m^{2}(s)$ as functions of $E=\sqrt{s}$ for two values of bare-mass $m_{0}(g g)=1600,1740 \mathrm{MeV}$, suggested by lattice calculations. The curves $\left(I_{0}\right)$ and $(I)$ are obtained by using $S U(3)_{f}$ singlet couplings and correspond to the undiagonalized and diagonalized cases respectively. The curves $\left(I I_{0}\right)$ and $(I I)$ are the analogs obtained by using the pattern of couplings found by the IBM group. Here there is negligible difference between the curves with $\gamma=1.1$ and 1.2. 


\begin{tabular}{|l|c|c|c|c|c|c|}
\hline Couplings & $m_{0}(g g)$ & $\Gamma_{\text {tot }}$ & $\Gamma_{\pi \pi}$ & $\Gamma_{K \bar{K}}$ & $\Gamma_{\eta \eta}$ & $\Gamma_{\eta \eta^{\prime}}$ \\
\hline \hline$S U(3)_{f}$ singlet & 1600 & 4 & 2 & 0.2 & 0.5 & 1.5 \\
\hline$S U(3)_{f}$ singlet & 1740 & 26 & 5 & 6 & 9 & 6 \\
\hline$I B M$ & 1600 & 25 & 0.5 & 1.5 & 21 & 2 \\
\hline$I B M$ & 1740 & 32 & 0.1 & 1 & 26 & 5 \\
\hline
\end{tabular}

Table 3: Widths of the glue-state to each single two pseudoscalar channel, for various choices of bare mass and couplings.

consistent analysis of data on all of peripheral and central production, $J / \psi$ radiative decays and $p \bar{p}$ annihilation [17] and show which of the predictions of Table III is in best agreement with experiment. Only then can one claim to have discovered the lightest glueball.

Acknowledgements. We would like to thank Frank Close and David Morgan for encouraging discussions and we acknowledge the support of the EURODA $\Phi$ NE Network grant ERBCMRXCT920026 of the EC Human and Capital Mobility program.

\section{References}

[1] J. Sexton et al., Phys. Rev. Lett.75, 4563 (1995).

[2] J. Sexton et al., presented at "Lattice 95", Melbourne, Australia, Jul 1995, hep-lat/9602022.

[3] G.S. Bali et al. (UKQCD), Phys. Lett. B 389, 378 (1993).

[4] C. Morningstar et al., presented at "Lattice 96", St. Louis, MO, Jun 1996, hep-lat/9608050.

[5] D.V. Bugg, Proc. of Hadron '89, Ajaccio, Corsica (ed. F. Binon et al), editions Frontiers, p.567. 
[6] Review of Particle Physics, Phys. Rev. D 54,30 (1996).

[7] N.A. Tornqvist, Z. Phys. C 68, 647 (1995).

[8] N. Isgur and J. Speth, Phys. Rev. Lett. 77, 2332 (1996);

M. Harada et al., Phys. Rev. Lett. (to be published), hep-ph/9609428.

[9] N.A. Tornqvist, M. Roos, Phys.Rev.Lett. 76,1575(1996).

[10] C. Amsler, F.E. Close, Phys. Rev. D 53, 295 (1996); Phys.Lett. B 353, 385 (1995).

[11] C. Amsler et al., Phys. Lett. B 342, 433 (1995); Phys. Lett. B 353, 571 (1995); Phys. Lett. B 355, 425 (1995).

[12] J.E. Augustin et al., Z. Phys. C 36, 369 (1987); Phys. Rev. Lett. 60, 2238 (1988); R.M. Baltrusaitis et al., Phys. Rev. D 35, 2077 (1987).

[13] S.J. Lindenbaum et al., Phys.Lett. B 274, 492 (1992).

[14] F. Binon et al., Nuovo Cimento 80, 363 (1984); D. Alde et al., Phys. Lett. B 201, 160 (1988).

[15] T.A. Armstrong et al., Phys. Lett. B 228, 536 (1989).

[16] As reported by R. Landua, Proc. Int. Conf. on HE Physics, Warsaw, 1996 (to be published).

[17] As in A.V. Sarantsev, Proc. of "Hadron 95", Manchester, (ed. M.C. Birse et al.), World Scientific, p. 384. 